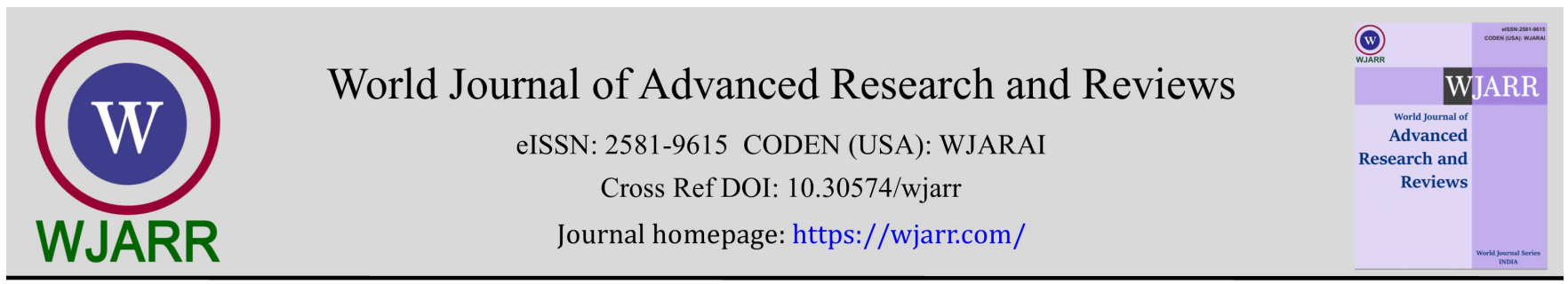

(RESEARCH ARTICLE)

\title{
Blood groups and COVID-19: Exploring the link
}

\author{
Syeda Kinza Bukhari ${ }^{1}$, Yusra Ashraf 2, Shahzeb Nasir ${ }^{3}$ and Kashif Akbar 4,* \\ 1 Trainee Medicine, Combined Military Hospital, Multan, and Punjab, Pakistan. \\ 2 District Headquarters Hospital, Rawalpindi, Punjab, Pakistan. \\ ${ }^{3}$ Army Medical College, Rawalpindi, Punjab, Pakistan. \\ ${ }^{4}$ Department of Commerce, Bahauddin Zakariya University, Multan, Punjab, Pakistan.
}

World Journal of Advanced Research and Reviews, 2021, 12(02), 267-272

Publication history: Received on 05 October 2021; revised on 08 November 2021; accepted on 10 November 2021

Article DOI: https://doi.org/10.30574/wjarr.2021.12.2.0596

\begin{abstract}
The SARS COVID-19 pandemic took the whole world by storm due to its high and rapid infectivity. As it spread, its patterns became clear, and it became evident that a few individuals were more predisposed to it than others. Showing a massive inflammatory response by the body, and a negative coagulative affect, doctors and scientists began to perceive the role of blood cells, and hence blood types in the disease process. This lead to the main objective of this research: to assess the link between COVID19 infection and blood group types, and follow disease outcome according to it. The study comprises data collected from patients within 3 hospital setting of Northern Pakistan, viz Military Hospital Rawalpindi, Benazir Bhutto Hospital Rawalpindi, and Pakistan institute of medical sciences Islamabad. The study was conducted during a period of 1 month i-e from 15th June 2020 to 15th July 2020 after approval by the worthy Ethical review committee of PEMH. The study design was Analytical- Case series. Purposive Sampling technique was used. The sample size was 306 based on WHO sample calculator. Patients who were PCR positive and symptomatic for COVID-19 were included whereas PCR negative and asymptomatic patients were excluded. SPSS version 25 was used to process the data and Graphs and Charts were used to explain it. Among 306 patients of COVID 19 the most commonly affected blood group was $\mathrm{A}+\mathrm{ve}$ followed by $\mathrm{B}+\mathrm{ve}$. The least affected blood group was $\mathrm{O}$ of which both positive and negative contributed to $17.71 \%$. A blood group contributed 36\%, while B 35\% and AB 10.8\%. The study substantiates that blood group A Rh positive has more susceptibility for SARS COVID-19 infection than other blood group types. Blood group 0 might have a protective effect against this infection.
\end{abstract}

Keywords: COVID-19; Blood Group Types; Coronavirus Pandemic; Healthcare

\section{Introduction}

The index case of severe acute respiratory syndrome Corona Virus 19 (COVID19) came to light in China in December 2019, and, soon reached a global scale, with a total of 148921 patients in Pakistan by July 2020 [1].

COVID19 is notorious for its high degree of infectivity and uncertain range of symptoms varying from fever, fatigue, myalgia, dry cough, anorexia, sputum production, pharyngitis, diarrhea, nausea, dizziness, headache, abdominal pain, or vomiting [2].

However, fever is not necessarily present with many patients having an unnoticeable low grade temperature from 37.5 to 38 degrees Celsius [3]. Less common symptoms included headache without any aura, and rhinorrhea, while many patients develop acute shortness of breath [4]. However not all patients with dyspnea go on to develop respiratory

\footnotetext{
${ }^{*}$ Corresponding author: Kashif Akbar

Department of Commerce, Bahauddin Zakariya University, Multan, Punjab, Pakistan.

Copyright $(2021$ Author(s) retain the copyright of this article. This article is published under the terms of the Creative Commons Attribution Liscense 4.0.
} 
distress or even require any ventilatory support [5]. According to the latest guidelines of the World Health Organization (WHO), COVID-19 hospital admission may only be given to those requiring oxygen supplementation [6].

To ensure adequate tertiary care, patients are divided into 3 broad categories based on the presence or absence of the symptoms mentioned above. The spectrum of disease ranges from category 'Mild', which includes patients who are COVID positive and with mild symptoms ( 1 or 2 of the above) but without any infiltrates on chest $x$-ray. The second category 'Moderate' include patients with less than $50 \%$ chest infiltration on $\mathrm{x}$-ray but without significant hypoxemia and respiratory rate $<20 / \mathrm{min}$, and requiring intermittent oxygenation. The last category is 'Severe' which includes patients with significant chest infection, hypoxemia, CRS, deranged coagulation factors, and cardiac involvement. (WHO treatment guidelines COVID19)

Increased severity of the disease is linked to a higher degree of involvement of the body's hemostatic system and thus some were more prone to it than others. Furthermore, a pattern was seen between those affected and their blood types. The prognosis of coronavirus disease (COVID-19) patients thus depends on their Blood types [7].

The term "blood group" refers to the entire system comprising red blood cell (RBC) antigens which usually play a role in transfusion reactions along with specific affinities to various diseases as described below. The ABO blood groups have a paramount role in hemostasis. They exert effects on plasma levels of Von Willibrand factor and factor VIII. Increased association of myocardial infarction, ischemic stroke, and venous thromboembolism is seen with blood groups A and $\mathrm{AB}[8]$.

Blood groups have been highly associated with some diseases in the past. Malaria in the African countries is selective against all non-O blood groups, while the deadly virus Small pox was selective against A blood type [9]. 0 blood type, is more prone to H. pylori induced peptic ulcer than any other type of blood group.9 Studies done in Wuhan, and the USA show a linkage of blood groups to the contraction of COVID19. Moreover, it is also demonstrated that blood group A was more frequent in patients who presented with severe pulmonary damage [10].

\section{Objective}

To find out the effect of blood group types on the risk of COVID-19 infection and its clinical outcome.

\section{Methodology}

This was an Analytical Case Series study carried out within 3 Tertiary Care Units of Rawalpindi viz Pakistan Emirates Military Hospital (PEMH), Benazir Bhutto Hospital (BBH), Pakistan Institute of Medical Sciences (PIMS). The Study was conducted over a period of 1-month i.e. from June 1st, 2020 to July 1st 2020 after approval by worthy ethical review committee of PEMH Rawalpindi. A Sample Size of 306 was calculated using WHO size calculator with 95\% confidence interval out of a sample of 1500 positive and symptomatic patients, with a 5\% Error. Patients were confirmed positive via real time PCR (company kit etc), and their blood samples were taken via aseptic ante-cubital venous sampling technique and analyzed via tube method. Nonprobability purposive sampling techniques were employed in this study. Data was collected by structured interview directly from patients or their attendants, after informed consent and full confidentiality, and the Data was analyzed using SPSS version 25. Frequencies and percentages that were collected were of categorical data.

\section{Results and discussion}

Out of the 306 participants admit in the hospitals during the study duration, 260 were male, and 46 were female contributing to a relative percentage of approximately $85 \%$ and $15 \%$ respectively. The patients selected were those who were positive and symptomatic, and thus their ages and various comorbidities had to be taken into consideration.

Co-morbids included Diabetes, Hypertension, Chronic kidney disease, ischemic heart disease, and a varying combination of these. Most patients were found to be without any comorbid diseases.

Relevant to this study each patient was assessed for blood group. The most commonly affected blood group was A +ve followed by B+ve. The least affected blood group was $\mathrm{O}$ of which both positive and negative contributed to $17.71 \%$. A blood group contributed $36 \%$, while B $35 \%$ and AB $10.8 \%$ 
World Journal of Advanced Research and Reviews, 2021, 12(02), 267-272

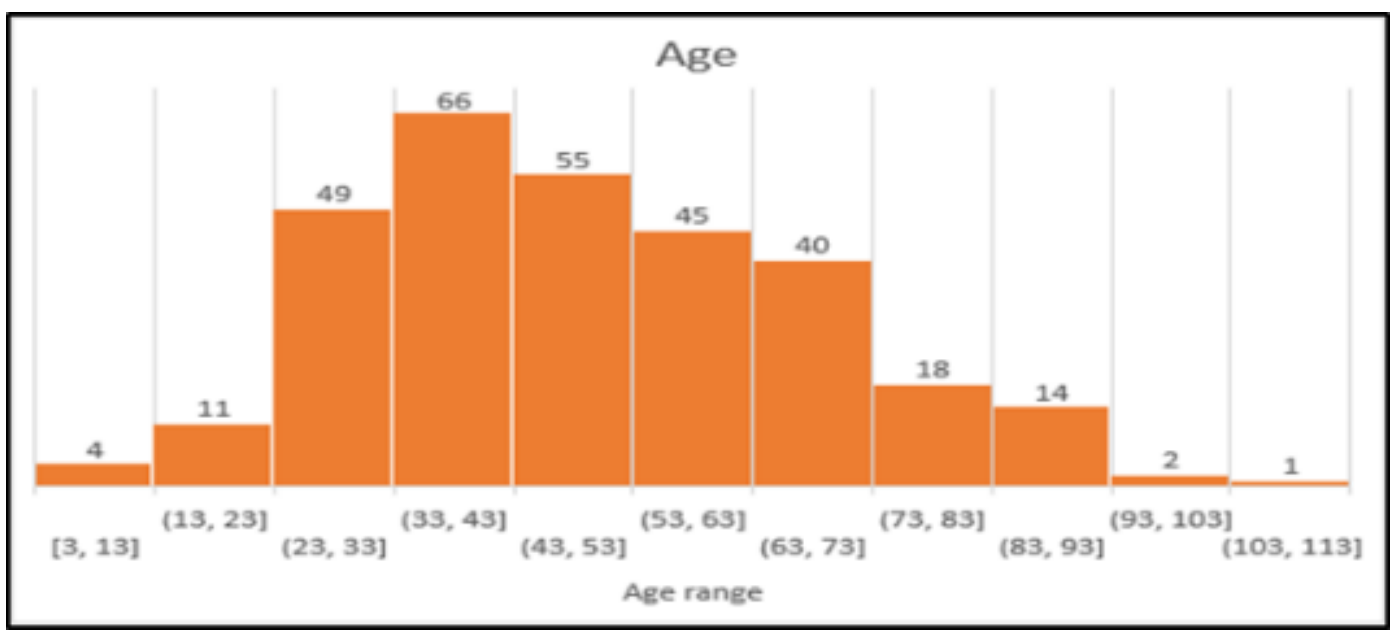

Figure 1 Age Range

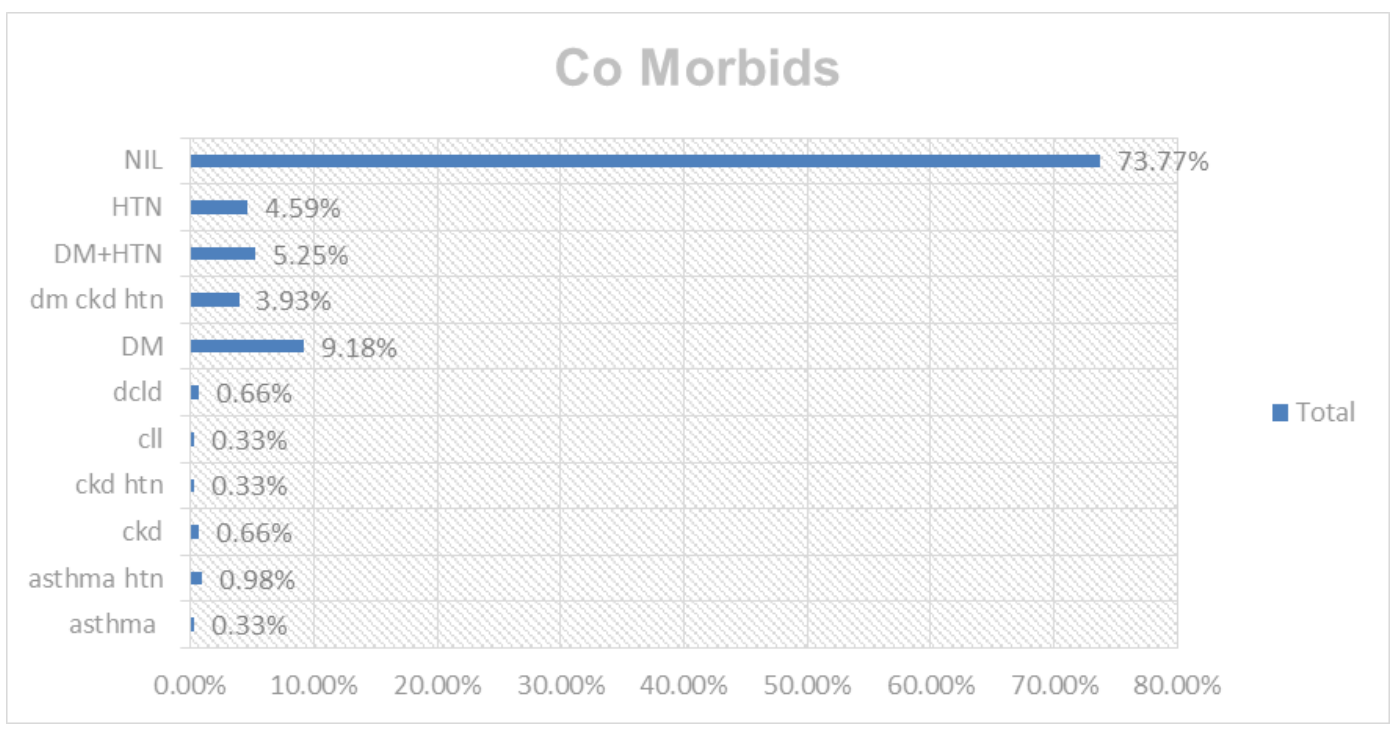

Figure 2 Co-Morbids

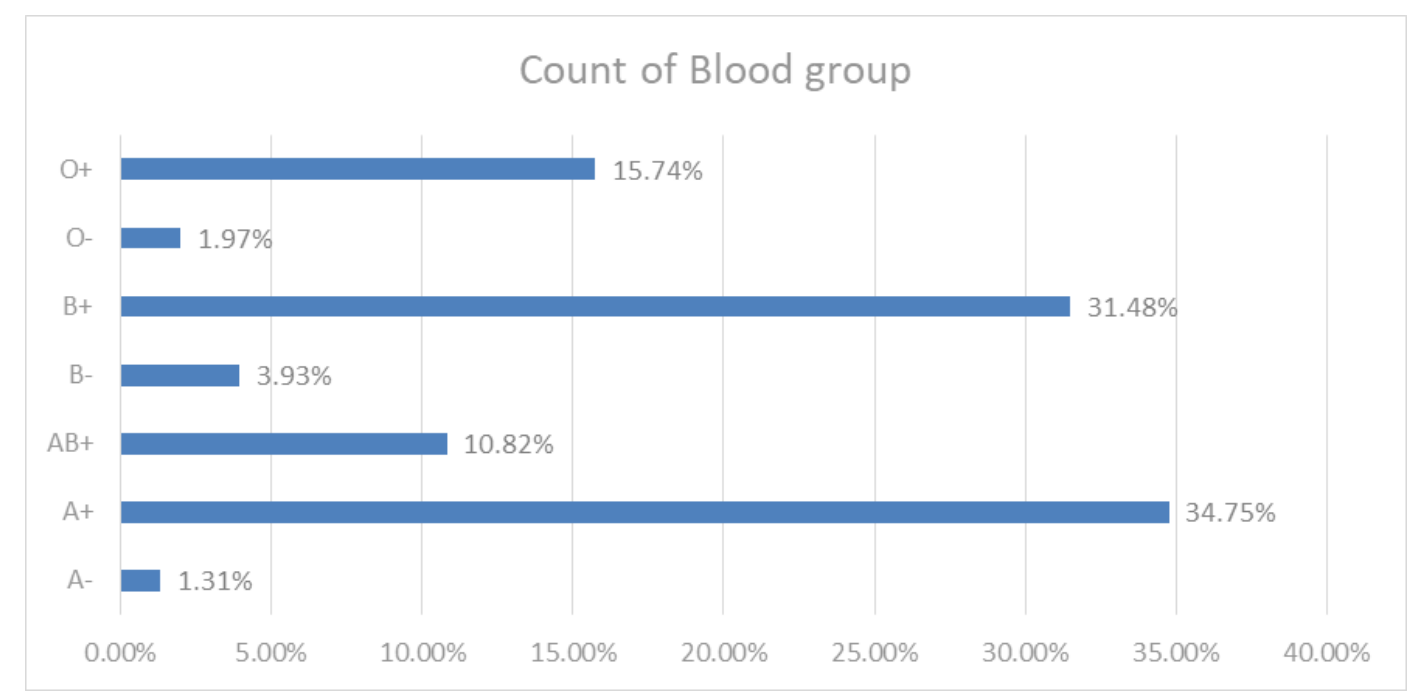

Figure 3 Count of Blood Group 
O+ve seemed to have a protective effect, to further analyze this effect, we decided to include the general effects of disease in each blood group. This prognosis was determined on the basis of 2 factors:

- Disease severity based on mild, moderate and severe.

- Method of artificial ventilation

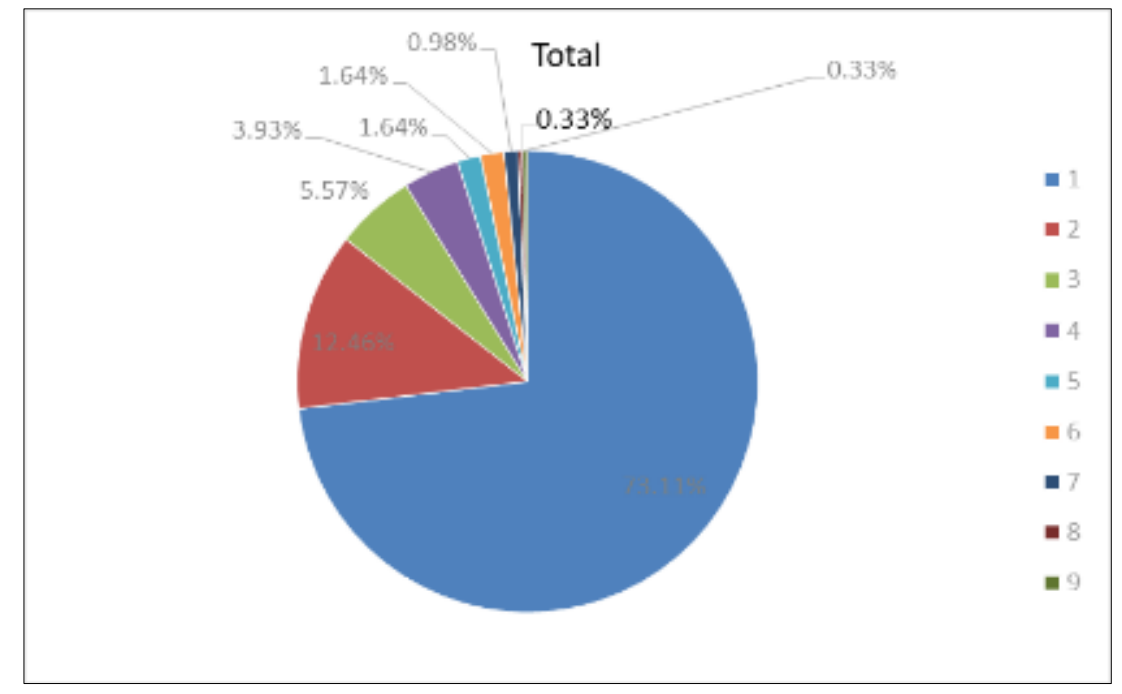

Figure 4 Prognosis

Out of the total 306 patients, most didn't require any form of oxygen support.15 ended up on ventilator, 36 received oxygen via Non Rebreather Mask. Seven were on CPAP whereas 24 received oxygen via Nasal prongs and Facemasks.

Out of the 38 on NRM, 20 (52\%) were A+ve, 4 (10\%) O+ve, 3 (7\%) A -ve, 1 (2\%) Ab+ve and 10 (26\%) were B+ve,

Whereas of the 17 on CPAP, 10 (58\%) were A+ve, $2(11 \%)$ 0+ve and $4(23 \%)$ were B+ve.

Consistently A+ve patients showed a poorer picture of corona disease severity and prognosis as compared to other blood groups, while 0 blood type showed promising improvement, and required less support.

The patients were broadly separated into mild, mod, severe, on the basis of clinical severity and comorbids.

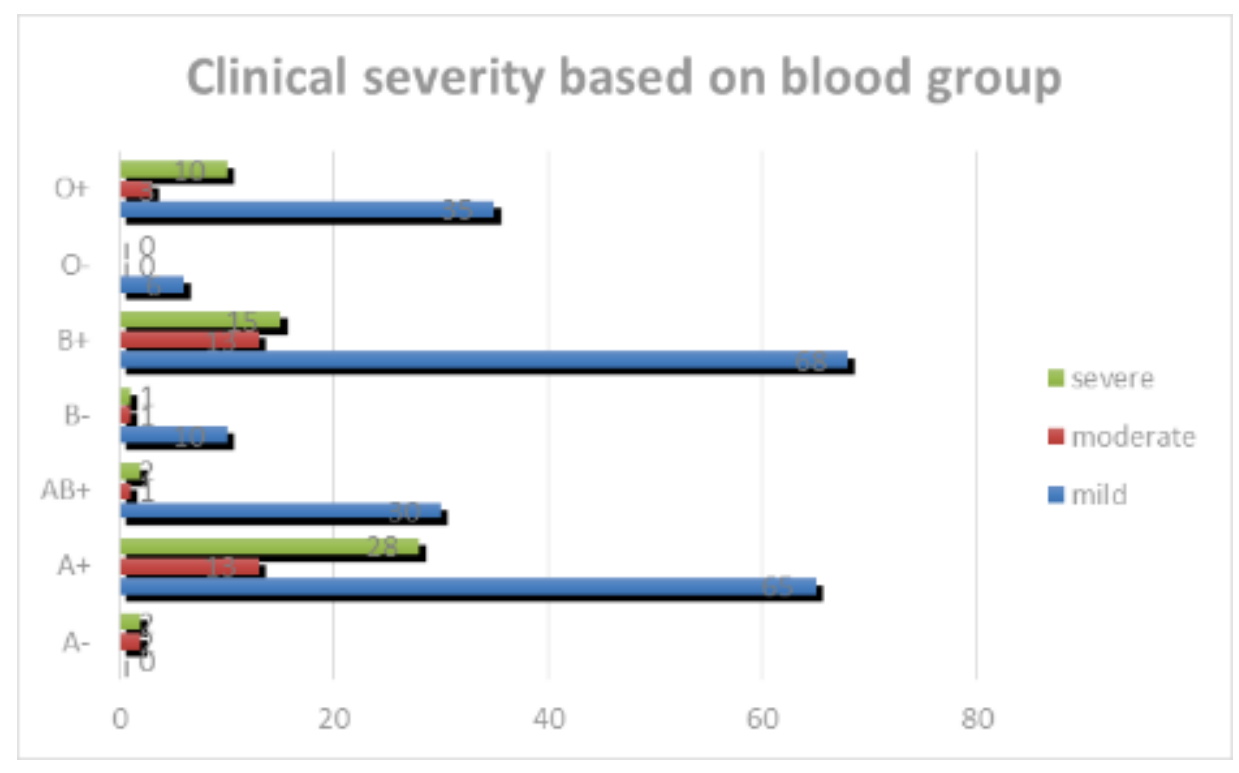

Figure 5 Clinical Severity Based on Blood Group 
As evident from the chart above, severity of COVID19 according to blood group revealed that most of the severe patients were A+ve. While the rest of the blood groups showed a milder course of disease. $13 \mathrm{~A}+\mathrm{ve}$ patients were severe as compared to 3 of $\mathrm{O}+\mathrm{ve}$.

One might think discrepancies may lie due to there being a naturally higher number of a particular blood group. But these were removed due to the following data:

A recent worldwide study [11] shows the following distribution of blood groups in our population: Blood groups 0 shows highest prevalence, followed by A.

Table 1 Distribution of Blood Groups

\begin{tabular}{|c|c|c|c|}
\hline $\mathbf{0}$ & $\mathbf{A}$ & $\mathbf{B}$ & $\mathbf{A B}$ \\
\hline $26.63 \%$ & $21.6 \%$ & $34.40 \%$ & $9.52 \%$ \\
\hline
\end{tabular}

This proves the significance of bare minimum amount of 0 positive COVID19 patients as compared to the fact that 0 positive blood group is actually present in high amounts in our population.

A total of 306 COVID-19 patients who met our inclusion criteria i.e. PCR positive and symptomatic from three corona center of northern Punjab were included in this study.

Among these 306 cases confirmed by PCR men had higher ratio than females or children. Whether this difference is behavioral or biological but men are affected in higher proportion as compared to female. Western Europe has also reported that $69 \%$ of the affected people were male. China and other countries have also showed a similar trend [7].The pathogenesis of severe COVID-19 and the respiratory failure associated with it is still unclear, but the comorbids especially older age and being male increase the chances of mortality [13].

In our study the most commonly affected blood group was $\mathrm{A}+$ followed by $\mathrm{B}+$ while blood group 0 , contributing $17.71 \%$ of the total.

Multiple studies conducted during this time have also shown that people with blood group A had a 45 percent increased chances of coronavirus infection and developing respiratory failure compared to people with other blood types. Whereas, people with blood group 0 had a 35 percent lower risk of developing severe COVID-19.

Genes responsible for blood types might play a role in the makeup of cell surfaces. The changes in cell-surface structures explain the vulnerability of certain blood types to COVID-19.14

The reason for this susceptibility probably lies in the connection between the proteins on the surface of Coronaviruses and carbohydrate groups on the surface cells of RBCs of different blood types. Individuals with blood group A have an extra sugar on the surface of their cells called anacitosal glucosamine, which is absent in those of blood group 0 [14].

However, when infected it was not only the blood group but the patient's own immunity that played an important role against the viral infection, this moreover determined the prognosis of disease in each individual.

Once a patient had contracted the disease, the role of attachment of the virus because of a specific blood group was complete. Now the body's infinite inflammatory response would take over and determine the course of disease, hence to our best understanding the clinical outcome was independent of the blood group.

Thus individuals with blood group A should be more cautious in this pandemic especially the health care workers who deal this virus on daily basis should take an extra care of themselves. Hospitals and the institutes should check their employee's blood group and guide them accordingly.

The limitation of our study is less number of participants and their individual clinical outcome. There should be more molecular studies to find out the exact cause of higher susceptibility in blood type A and protective effect of blood type 0 . 


\section{Conclusion}

The Results of this study suggested that blood group A; Rh+ive have increased susceptibility to COVID-19 infection while blood group 0 both $\mathrm{Rh}+$ ive and Rh-ive have a protective role against COVID-19 infection. The Blood Group types, does not have a strong link with clinical outcome of a patient infected by SARS COVID-19.

\section{Compliance with ethical standards}

\section{Acknowledgments}

The Authors sincerely appreciate the support of Healthcare Departments from Pakistan. They helped the authors to make this research possible.

\section{Disclosure of conflict of interest}

None.

\section{Statement of informed consent}

Informed consent was obtained from all individual participants included in the study.

\section{References}

[1] Lai CC, Shih TP, Ko WC, et al. Severe acute respiratory syndrome coronavirus 2 (SARS-CoV-2) and corona virus disease-2019 (COVID-19): the epidemic and the challenges. International journal of antimicrobial agents. $17 \mathrm{Feb}$ 2020: 105924.

[2] Ortiz-Prado E, Simbaña-Rivera K, et al. Clinical, molecular, and epidemiological characterization of the SARS-CoV2 virus and the Coronavirus Disease 2019 2020; 98(1): 115094.

[3] Huang C., Wang Y., Li X., et al. Clinical features of patients infected with 2019 Novel Coronavirus in Wuhan. China the Lancet. 2020; 395: 497-506.

[4] Xu X.W., Wu X.X., et al. Clinical findings in a group of patients infected with the 2019 Novel Coronavirus (SARSCov-2) outside of Wuhan, China: Retrospective case series. The BMJ. 2020; 368: 1-7.

[5] Wang D., Hu B., et al. Clinical characteristics of 138 hospitalized patients with 2019 Novel Coronavirus-infected pneumonia in Wuhan, China. JAMA - J Am Med Assoc. 2020; 1-9.

[6] Who W. WHO-2019-nCoV-clinical-2020.4-eng. 2020; 1-21.

[7] Angela Betsaida B. Laguipo, BSN [Internet]. 2020 [updated 2020 Jun 18; cited 2021 Nov 11]. Available from https://www.news-medical.net/amp/news/20200618/Blood-types-and-COVID-19-risk-confirmed.aspx

[8] Mitra R, Mishra N. Blood groups systems. Indian journal of anaesthesia. Sep 2014; 58(5): 524.

[9] Anstee D. The relationship between blood groups and disease. Blood. 10 Jun 2010; 115(23): $4635-43$.

[10] Göker H, Karakulak EA, et al. The effects of blood group types on the risk of COVID-19 infection and its clinical outcome. Turkish journal of medical sciences. 23 Jun 2020; 50(4): 679-83.

[11] Rhesus Negative.

[12] Byrne KM, Byrne PC. Other blood group systems- IMMUNOHEMATOLOGY-WASHINGTON DC-. 2004; 20(1): 508.

[13] Clary Estes, Former Contributor, Healthcare [Internet]. 2020 [updated Mar 2020; cited 2021 Nov 11]. Available from https://www.forbes.com/sites/claryestes/2020/03/20/what-the-relationship-between-blood-type-andcoronavirus-susceptibility-means-for-future-treatments/\#2a6764f677c8

[14] WEBMD ARCHIVES [Internet]. 2020 [updated March 2020; cited 2021 Nov 11]. Available from https://www.webmd.com/lung/news/20200320/blood-type-may-affect-COVID19-risk-study. 